\title{
Ligand exchange on the surface of cadmium telluride quantum dots with fluorosurfactant-capped gold nanoparticles: Synthesis, characterization and toxicity evaluation
}

\author{
Lingyun Wang ${ }^{\mathrm{a}}$, Hongxia Zhang ${ }^{\mathrm{a}}$, Chao Lu ${ }^{\mathrm{a}, *}$, Lixia Zhao ${ }^{\mathrm{b}}$ \\ a State Key Laboratory of Chemical Resource Engineering, Beijing University of Chemical Technology, Beijing 100029, China \\ ${ }^{\mathrm{b}}$ State Key Laboratory of Environmental Chemistry and Ecotoxicology, Research Center for Eco-Environmental Sciences, Chinese Academy of Sciences, Beijing 100085, China
}

\section{A R T I C L E I N F O}

\section{Article history:}

Received 18 June 2013

Accepted 17 September 2013

Available online 29 September 2013

\section{Keywords:}

Quantum dots

Gold nanoparticles

Ligand exchange

Thioglycollic acid

Citrate

Cytotoxicity

\begin{abstract}
A B S T R A C T
CdTe quantum dots (QDs) can provide high-intensity and photostable luminescent signals when they are used as labeling materials for sensing trace amounts of bioanalytes. However, a major concern is whether the capping ligands of CdTe QDs cause toxic effects in living systems. In the current study, we address this problem through the complete ligand transformation of CdTe QDs from toxic thiolglycolic acid (TGA) to green citrate, which is attributed to the $\mathrm{Cd}-\mathrm{S}$ bond breaking and the $\mathrm{Au}-\mathrm{S}$ bond formation. The highly efficient depletion of $\mathrm{S}$ atom from the surface of the CdTe QDs occurs after the addition of fluorosurfactant (FSN)-capped gold nanoparticles into TGA-capped CdTe QDs, accompanying with the rapid aggregation of FSN-capped gold nanoparticles via noncrosslinking mechanism in the presence of high salt. After the ligand transformation, negligible differences are observed on both photoluminescence spectra and luminescent quantum yield. In addition, the cytotoxicity of the original and new-born CdTe QDs is detected by measuring cell viability after the nanoparticle treatment. In comparison with the original TGA-capped QDs, the new-born CdTe QDs can induce minimal cytotoxicity against human hepatocellular liver carcinoma (HepG2) cells even at high dosages. Our study indicates that the extremely simple method herein opens up novel pathways for the synthesis of green CdTe QDs, and the as-prepared citrate-capped CdTe QDs might have great potential for biological labeling and imaging applications.
\end{abstract}

(c) 2013 Elsevier Inc. All rights reserved.

\section{Introduction}

CdTe quantum dots (QDs) have received great attention for both fundamental research and potential applications due to narrow emission band, broad absorption, and high fluorescence quantum yields [1-3]. CdTe QDs can be synthesized by a non-aqueous organometallic route at high temperature (e.g., $300^{\circ} \mathrm{C}$ ) or an aqueous method at $100{ }^{\circ} \mathrm{C}[4-6]$. The synthesized QDs through nonaqueous organometallic routes usually need to undergo surface exchange with hydrophilic ligands in aqueous solution for biological applications. Over the last decade, the ligand exchange procedures of quantum dots have been significantly improved [7]. On the other hand, the preparation of water soluble QDs with longterm colloidal stability and high quantum yields has been well demonstrated using thiol as capping ligands, such as 3-mercaptopropionic acid (MPA) and thiolglycolic acid (TGA) [5]. However, it is noteworthy that these thiol ligands are volatile liquid with an awful odor and their carcinogenic property further deters their use from laboratories under stringent safety requirements.

\footnotetext{
* Corresponding author.

E-mail address: luchao@mail.buct.edu.cn (C. Lu).
}

Furthermore, it recognizes that the cytotoxicity of CdTe QDs is dependent on the surface ligands rather than on the core material, and thus surface modification of CdTe QDs have an important effect on cytotoxicity $[8,9]$. In conclusion, it still remains with primary importance to explore environment-friendly synthetic routes for high-quality aqueous CdTe nanocrystals.

Recently, it has been reported that citrate can act as a green capping agent to substitute TGA or MPA for the synthesis of watersoluble QDs [10-13]. However, citrate is a relatively weak stabilizer bound to the surface of colloidal QDs, which may suffer from a decrease in quantum yield to a certain degree. In addition, the colloidal stability of citrate-capped QDs would be sensitive to $\mathrm{pH}$ and salt concentration. Therefore, the citrate-capped QDs might have little potential for biological labeling and imaging applications. On the other hand, there have been several researches reported on the great improvement of the ligand exchange procedures [14-16]. In this study, we are motivated to explore the possibility of TGA ligand transformation of CdTe QDs without affecting luminescent characteristics of the QDs to meet the future's demand for nanotoxicity studies and bioapplications.

It is now well-known that thiol ligands are considered to show a high affinity to noble metal surfaces via an $\mathrm{Au}-\mathrm{S}$ bond. In recent 
years, thiol compounds have been used to modify gold nanoparticles to improve their stability, dispersibility, and biocompatibility, triggered by potential applications in different branches of nanotechnology such as sensing, electronic devices, catalysis and biomedical applications [17-20]. On the other hand, thiol compounds (e.g., cysteine and homocysteine) can induce the aggregation of gold nanoparticles via interparticle crosslinking mechanism or noncrosslinking mechanism, which could be used for colorimetric sensing of thiol compounds [21]. Nonionic fluorosurfactants (Zonyl FSN) are commercially available with a polyoxyethylene chain in their hydrophilic part and a fluorocarbon chain at the hydrophobic part. FSN ligands can be attached to the surface of gold nanoparticles via physical interaction between its hydroxyl group at the end of the hydrophilic part and gold [22]. In 2007, $\mathrm{Zu}$ group pioneered in the use of the distance-dependent optical properties of FSN-capped gold nanoparticles for selective colorimetric detection of aminothiols (cysteine and homocysteine) via noncrosslinking mechanism in the presence of high salt [23]. Subsequently, the FSN-capped gold nanoparticles have been extensively used as colorimetric probes for a variety of aminothiols and DNA [24-27]. These interesting reports inspire us to extend the application of FSN-capped gold nanoparticles in the field involving in thiol compounds.

In this study, we reported a simple method to transform capping ligands of CdTe QDs from toxic TGA to green citrate, allowing to retain sufficiently strong luminescence of CdTe nanocrystals. The procedure was schematically described in Fig. 1: a certain amount of TGA-capped CdTe QD was mixed with an appropriate volume of the FSN-capped gold nanoparticles in the presence of phosphate buffer solution (PBS). TGA ligands were removed from the surface of CdTe QDs attributing to the $\mathrm{Cd}-\mathrm{S}$ bond breaking and the $\mathrm{Au}-\mathrm{S}$ bond formation. As a result, the aggregation of the FSN-capped gold nanoparticles occurred via noncrosslinking mechanism in the presence of high salt. The ligand transformation mechanism was demonstrated by UV-visible spectroscopy, powder X-ray diffraction (XRD) measurements, transmission electron microscopy (TEM) images, energy dispersive X-ray spectroscopy (EDX) and Fourier transform infrared (FT-IR) spectrum. In addition, the cytotoxicity of the as-prepared citrate-capped CdTe QDs on HepG2 cells was compared to that of the TGA-capped CdTe QDs. The results showed the as-prepared citrate-capped CdTe QDs can induce minimal cytotoxicity even at high dosages, which might be of interest for biological labeling and imaging applications.

\section{Experimental}

\subsection{Reagents}

All reagents were of analytical grade and used without further purification. All solutions were prepared with deionized water (Milli Q, Millipore, Barnstead, CA, USA). Zonyl FSN-100 ( $\mathrm{F}\left(\mathrm{CF}_{2-}\right.$ $\left.\left.\mathrm{CF}_{2}\right)_{1-7} \mathrm{CH}_{2} \mathrm{CH}_{2} \mathrm{O}\left(\mathrm{CH}_{2} \mathrm{CH}_{2} \mathrm{O}\right)_{0-15} \mathrm{H}\right)$ and $\mathrm{MTT}$ (3-(4,5-dimethylthiazol-2-yl)-2,5-diphenyltetrazolium bromide) were supplied by Sigma-Aldrich (St. Louis, USA). Hydrogen tetrachloroaurate (II) trihydrate $\left(\mathrm{HAuCl}_{4} \cdot 3 \mathrm{H}_{2} \mathrm{O}\right)$, MPA and trisodium citrate were purchased from Acros (Geel, Belgium). The pH of PBS was adjusted with $\mathrm{NaOH}$ or $\mathrm{HCl}$. Ethanol, Dimethyl sulfoxide (DMSO), $\mathrm{NaOH}$, $\mathrm{Na}_{2} \mathrm{HPO}_{4} \cdot 12 \mathrm{H}_{2} \mathrm{O}$ and $\mathrm{Pb}\left(\mathrm{NO}_{3}\right)_{2}$ were obtained from Beijing Chemical Reagent Company. Tellurium powder (Te, 99.999\%), TGA, $\mathrm{CdCl}_{2}$, $\mathrm{NaH}_{2} \mathrm{PO}_{4}$ and $\mathrm{NaBH}_{4}$ were obtained from Tianjin Chemical Reagent Company. Human hepatocellular liver carcinoma (HepG2) cells were provided by the Institute of Basic Medical Sciences Chinese Academy of Medical. Dulbecco's modified Eagle's medium (DMEM, Gibco, NY, USA) was purchased from Gibco (Invitrogen, USA) supplemented with $10 \%$ fetal bovine serum (FBS, Hyclone) and $1 \%$ penicillin/streptomycin (Hyclone).

\subsection{Apparatus}

The UV-visible spectra of the FSN-capped gold nanoparticles were acquired on a Shimadzu UV-3600 spectrophotometer (Tokyo, Japan). Fluorescence (FL) spectra were collected with a Hitachi F7000 fluorescence spectrophotometer (Tokyo, Japan). The excitation slit and the emission slit were maintained at $5.0 \mathrm{~nm}$ and $10.0 \mathrm{~nm}$, respectively. The scan rate of the monochromators was maintained at $2400 \mathrm{~nm} / \mathrm{min}$. The excitation wavelength was set at $390 \mathrm{~nm}$. The EDX data and the sizes of CdTe QDs were confirmed through TEM measurements using Tecnai $\mathrm{G}^{2} 20$ TEM (FEI, USA) at an accelerating voltage of $200 \mathrm{kV}$. TEM specimens were prepared by depositing an appropriate amount of CdTe QDs onto the carbon-coated copper grid, and the excess solution was wicked away by a filter paper. The grid was subsequently dried in air before measure. Thermo Electron Nicolet 6700 FT-IR spectrometer (Madison, WI, USA) was used to record the spectrum of CdTe QDs. XRD patterns of the CdTe QDs were performed on a Bruker (Germany) D8 ADVANCE X-ray diffractometer equipped with graphite-monochromatized $\mathrm{Cu} \mathrm{K} \alpha$ radiation

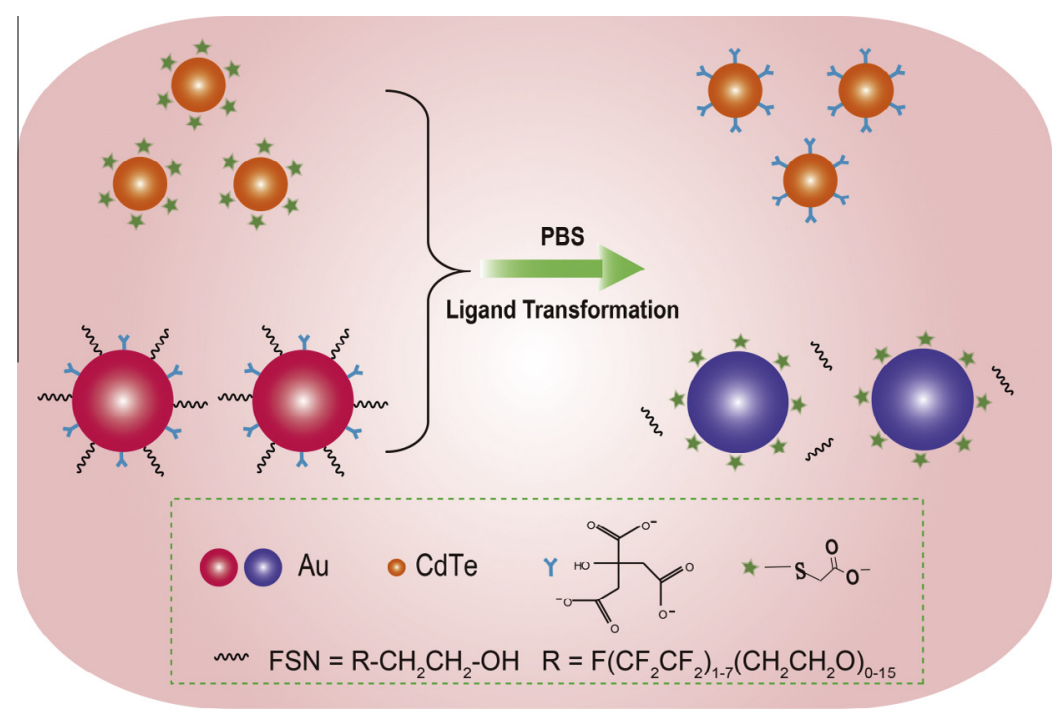

Fig. 1. Schematic illustration of ligand transformation mechanism between FSN-capped gold nanoparticles and TGA-capped CdTe QDs. 
( $\lambda=1.5406 \AA$ ). The $2 \theta$ angle of the diffractometer was stepped from $2^{\circ}$ to $70^{\circ}$ at a scan rate of $10^{\circ} / \mathrm{min}$. The centrifugation was operated on a TGL-16B centrifugal machine (Shanghai Anting Scientific Instrument Factory, Shanghai, China). The HepG2 cells were incubated in a $\mathrm{CO}_{2}$ incubator (Napco, Model 5410), and observed with an inverted microscope (Olympus 1X71, Tokyo, Japan). In MTT experiment, constant temperature microporous fast oscillator (QB9006, China) and a microplate reader (Synergy5, BioTek, USA) were used.

\subsection{Synthesis and characterization of TGA-capped CdTe QDs}

All glassware for the preparation of TGA-capped CdTe QDs was thoroughly cleaned with freshly prepared $\mathrm{HNO}_{3}$ aqueous solution, rinsed extensively with ultrapure water, and then dried in an oven at $100{ }^{\circ} \mathrm{C}$ for $2-3 \mathrm{~h}$. CdTe QDs were synthesized according to the literature procedure with little modification $\left(\mathrm{Cd}^{2+}: \mathrm{Te}^{2-}: \mathrm{TGA}=1: 0.5: 2.5\right)$ [28]. Briefly, TGA was firstly injected into nitrogen-saturated $\mathrm{CdCl}_{2}$ aqueous solution, followed by adjusting the $\mathrm{pH}$ value of the mixed solution to 11.5 with $2.0 \mathrm{M} \mathrm{NaOH}$. Then the freshly prepared NaHTe precursor by the reaction between Te powder and $\mathrm{NaBH}_{4}$ under $\mathrm{N}_{2}$ atmosphere at $60^{\circ} \mathrm{C}$ was added to the above mixture solution. Finally, the solution was then heated and refluxed under nitrogen flow at $96{ }^{\circ} \mathrm{C}$. The concentration of CdTe QD was calculated (14.3 $\left.\mu \mathrm{M}\right)$ according to the literature [29], where the extinction coefficients at particular wavelength can be estimated from the average TEM size. The CdTe QD size was $2.7 \mathrm{~nm}$ according to the following expression: $D=\left(9.8127 \times 10^{-7}\right) \lambda^{3}-\left(1.7147 \times 10^{-3}\right) \lambda^{2}+(1.0064) \lambda-194.84$, where $D(2.7 \mathrm{~nm})$ was the size of CdTe QDs, and $\lambda(518 \mathrm{~nm})$ was the wavelength of the absorption peak of CdTe QDs [6]. To purify the TGA-capped CdTe QDs, the free $\mathrm{CdCl}_{2}$ and TGA were removed via dialysis for $24 \mathrm{~h}$ in $0.01 \mathrm{M} \mathrm{NaOH}$ solution by the dialysis membrane with a molecular weight of cutoff 7000 . After the ligand transformation was finished between the FSN-capped gold nanoparticles and TGA-capped CdTe QDs, the citrate-capped CdTe QDs were obtained using a simple centrifuge to remove the aggregated gold nanoparticles.

The fluorescence quantum yields $\left(\Phi_{x}\right)$ of the TGA-capped CdTe QDs and new-born CdTe QDs were calculated according to the following formula: $\Phi_{x}=\left[\Phi_{\text {std }} \cdot F \cdot A_{\text {std }} \cdot n_{\text {std }}^{2}\right] /\left[F_{\text {std }} \cdot A \cdot n_{\text {std }}^{2}\right]$, where $F$ and $F_{\text {std }}$ are the areas under the fluorescence curves for sample and standard, respectively; $A$ and $A_{\text {std }}$ are the absorbances of the sample and standard at the excitation wavelength, respectively, while $n$ and nstd are the refractive indices of solvents in which the sample and standard are dissolved, respectively. The fluorescence quantum yield of Rhodamine 6G standard solution in ethanol $\left(\Phi_{\text {std }}\right)$ was 0.95 .

\subsection{Synthesis of FSN-capped gold nanoparticles}

All glassware for preparation of gold nanoparticles was thoroughly cleaned with freshly prepared aqua regia $\left(\mathrm{HNO}_{3}: \mathrm{HCl}=1: 3\right)$, rinsed extensively with deionized water, and then dried in an oven at $100{ }^{\circ} \mathrm{C}$ for $2-3 \mathrm{~h}$. The $14 \mathrm{~nm}$ FSN-capped gold nanoparticles were prepared following the literature procedure [22]. Briefly, $50 \mathrm{~mL}$ solution of $0.04 \%$ sodium citrate was brought to a vigorous boil with stirring in a round-bottom flask with a reflux condenser, and then $85 \mu \mathrm{L}$ of $5 \% \mathrm{HAuCl}_{4}$ was added to the stirring and refluxing sodium citrate solution. The solution was maintained at the boiling point with continuous stirring for $15 \mathrm{~min}$. After the solution was cooled to room temperature with continuous stirring, $0.4 \mathrm{~mL}$ of $5 \%$ FSN-100 was added. The suspension was stored at $4{ }^{\circ} \mathrm{C}$ until further use. Moreover, spherical gold nanoparticles were prepared by varying the concentration of citrate during the reduction step. Assuming spherical particles with density equivalent to that of bulk gold $\left(19.30 \mathrm{~g} \mathrm{~cm}^{-3}\right)$, the concentration of the $14-, 30-, 42-$, and 56-nm FSN-capped gold nanoparticles was calculated to be ca. $\sim 2.9,0.29,0.11$ and $0.05 \mathrm{nM}$, respectively [22].

\subsection{Pretreatment of HepG2 cells}

HepG2 cells were isolated from human, and then are grown in vitro to investigate the cytotoxicity of QDs. HepG2 cells were cultured in high glucose DMEM supplemented with 10\% FBS and $1 \%$ antibiotics (penicillin/streptomycin, $100 \mathrm{U} / \mathrm{mL}$ ). The HepG2 cells were kept in a $37^{\circ} \mathrm{C}, 5 \% \mathrm{CO}_{2}$ humidified incubator, and then were seeded in 96 -well plates at a density of $5 \times 10^{4}$ cells $/ \mathrm{mL}$. Once the dishes became confluent by $70-80 \%$, the TGA- or citrate-capped CdTe QDs were added to it and incubated for $19 \mathrm{~h}$ and the cells images were obtained with an inverted microscope. Cells were incubated in medium containing $5.0 \mathrm{mg} / \mathrm{mL}$ of MTT for $4 \mathrm{~h}$. Thereafter, the unreacted MTT solution was removed by centrifugation and the precipitated violet crystals (formazan) were redissolved in $100 \mu \mathrm{L}$ DMSO on the shaking tables for $10 \mathrm{~min}$ at low speed. Finally, the absorbance at $490 \mathrm{~nm}$ was measured using a BioTek microplate reader. The HepG2 cells viability (\%) was evaluated as follows: cells viability $(\%)=\left(A_{\text {test }} / A_{\text {control }}\right) \times 100 \%$, where $A_{\text {test }}$ stood for the absorbance of formazan obtained from the cells cultured with CdTe in individual well and $A_{\text {control }}$ was defined in a similar way of the blank experiments. The treatment for the control group HepG2 cells was the same as described above, except that no CdTe QDs was added to the medium.

\section{Results and discussion}

\subsection{Transformation of TGA from CdTe QD to FSN-capped gold nanoparticles}

The molar extinction coefficient of the gold nanoparticles $\left(1.76 \times 10^{8} \mathrm{~L} / \mathrm{mol} \mathrm{cm}\right.$ at $\left.\lambda_{\max }=517 \mathrm{~nm}\right)$ [30] is much greater than that of CdTe QDs $\left(7.32 \times 10^{4} \mathrm{~L} / \mathrm{mol} \mathrm{cm}\right.$ at $\left.\lambda_{\max }=518 \mathrm{~nm}\right)$ [31]. Therefore, according to Beer's Law, which states that $\mathrm{A}=\varepsilon_{\lambda} \mathrm{bc}$, the absorption spectra of the mixtures are dominated by the gold nanoparticles. In addition, it is well-known that a small amount of the $14 \mathrm{~nm}$ gold nanoparticles can bind lots of thiol groups (thiol/gold nanoparticle $=4000$ ) via stronger $\mathrm{Au}-\mathrm{S}$ bonds $[32,33]$. In theory, $12 \mu \mathrm{M}$ TGA can be attached to the surface of the $14 \mathrm{~nm}$ gold nanoparticles ( $2.9 \mathrm{nM})$. Therefore, such a small amount of the FSN-capped gold nanoparticles is enough to fully replace TGA of the QDs. In this study, the $100 \mu \mathrm{L}$ as-prepared TGA-capped CdTe QDs was mixing with $1.0 \mathrm{~mL} \mathrm{14-,} \mathrm{30-,} \mathrm{42-,} \mathrm{and} \mathrm{56-,} \mathrm{nm}$ FSN-capped gold nanoparticles in $20 \mathrm{mM}$ pH 8.0 PBS (see Fig. S1 in Supplementary information). Note that the addition of $20 \mathrm{mM}$ $\mathrm{pH}$ 8.0 PBS in the colloidal solution led to immediate aggregation of the gold nanoparticles stabilized electrostatically by adsorbed citrate (without FSN modification), indicating that the FSN ligands played a key role in the ligand exchange procedures of the QDs. Interestingly, as shown in Fig. 2, the absorption band at $520 \mathrm{~nm}$ decreased while new bands appeared at longer wavelengths, indicating that the gold nanoparticles aggregated as a result of the selfassembly of TGA on the surface of gold nanoparticles. In addition, TEM images clearly showed the formation of gold aggregation, thereby resulting in red-to-blue ${ }^{1}$ color change via noncrosslinking mechanism (Fig. 2). The rapid aggregation resulted from the TGA-coated gold nanoparticles was ascribed to the dissociation of FSN ligands from the gold nanoparticle surfaces after the ligand transformation, destroying the excellent gold colloidal stability in the presence of high salt concentrations as a result of the relatively

\footnotetext{
${ }^{1}$ For interpretation of color in Fig. 2, the reader is referred to the web version of this article.
} 


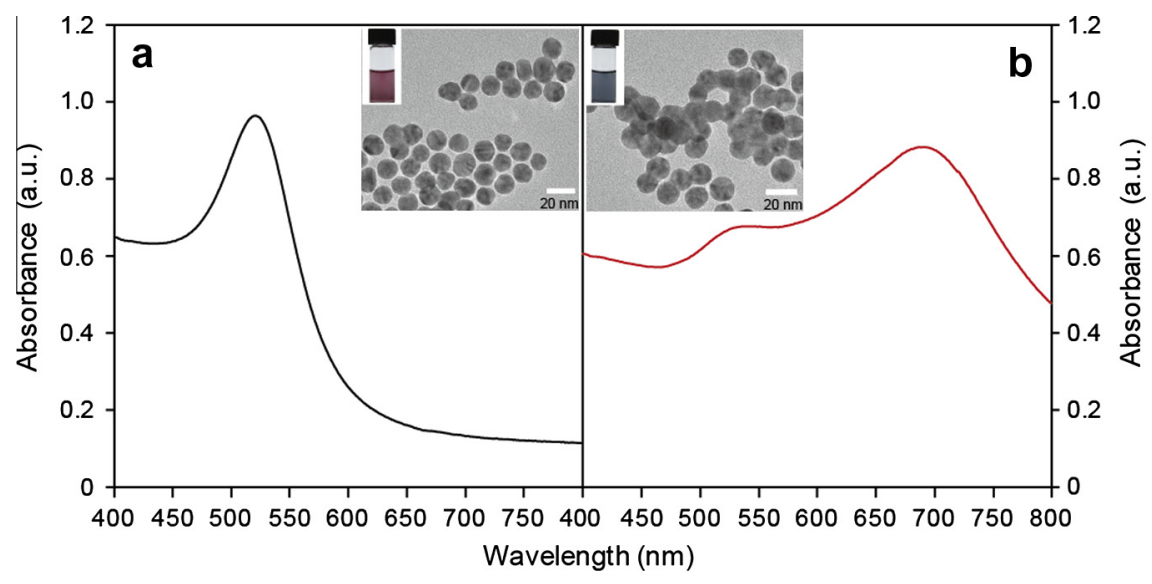

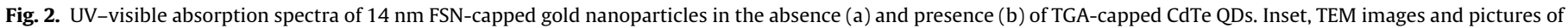
FSN-capped gold nanoparticles in the absence/presence of TGA-capped CdTe QDs.

larger steric hindrance of FSN ligands [23]. However, little spectral change occurred when the as-prepared TGA-capped CdTe QDs was mixing with the FSN-capped gold nanoparticles in the presence of low salt (2.0 mM PBS). The incubation time of the mixing solution was investigated at room temperature with gentle stirring. The results showed that a redshift of the absorption band of the gold nanoparticles was observed with increasing the incubation time until $30 \mathrm{~min}$ (Fig. S2). Therefore, $30 \mathrm{~min}$ was selected as the optimum time in our experiments. In addition, the aggregation of the citrate-capped gold nanoparticles occurred as a result of electrostatic interaction between carboxyl groups and $\mathrm{Pb}^{2+}$ [34] (Fig. S3a). However, the FSN-capped gold nanoparticles can keep stable in the presence of $50 \mu \mathrm{M} \mathrm{Pb}^{2+}$ [35] (Fig. S3b). Interestingly, when $50 \mu \mathrm{M} \mathrm{Pb}^{2+}$ was added into the FSN-capped gold nanoparticles after the reaction of the CdTe QDs, the absorption spectrum of the gold colloidal solution exhibited a red shift in the peak wavelength (Fig. S3c). We reasoned that the carboxyl groups of TGA exposed on the outer surface of the gold nanoparticles are able to interact with $\mathrm{Pb}^{2+}$ via interparticle crosslinking mechanism [36] (Fig. S4). As a result, the experiment of $\mathrm{Pb}^{2+}$ induced aggregation can directly confirm the TGA transformation of CdTe QDs from the surface of the QDs to gold nanoparticles.

\subsection{Complete depletion of TGA from the surface of CdTe QDs}

The driving force in interchanging reaction was strong $\mathrm{Au}-\mathrm{S}$ covalent bond between gold nanoparticles and TGA, which made the possibility of exchange of TGA from the surface of CdTe. In this study, EDX data of the TGA-capped CdTe QDs were investigated before and after addition of the FSN-capped gold nanoparticles into the TGA-capped CdTe colloidal solutions. To avoid a possible effect of the sampling location, EDX spectra were collected for at least twenty measurements at randomly chosen positions of each sample. Fig. 3 revealed that a complete depletion of $\mathrm{S}$ atom from the surface of the CdTe QDs occurred after the addition of the FSNcapped gold nanoparticles, which suggested that TGA can be strongly attached to the surface of the FSN-capped gold nanoparticles via the $\mathrm{Au}-\mathrm{S}$ bonds $[37,38]$. Note that the signal of phosphorus element appeared after the ligand exchange, which was attributed to the presence of phosphate in the new-born CdTe QD solution. Furthermore, we compared the EDX spectra of gold nanoparticles before and after mixing with TGA-capped CdTe QDs. The results showed EDX peaks of Au and S were almost at the same location, which were consistent with the published papers $[39,40]$.

FT-IR spectra were employed to evaluate the differences of capping reagents between the TGA-capped and new-born CdTe QDs. As seen in Fig. 4, the peaks of $3431 \mathrm{~cm}^{-1}$ and $3440 \mathrm{~cm}^{-1}$ were

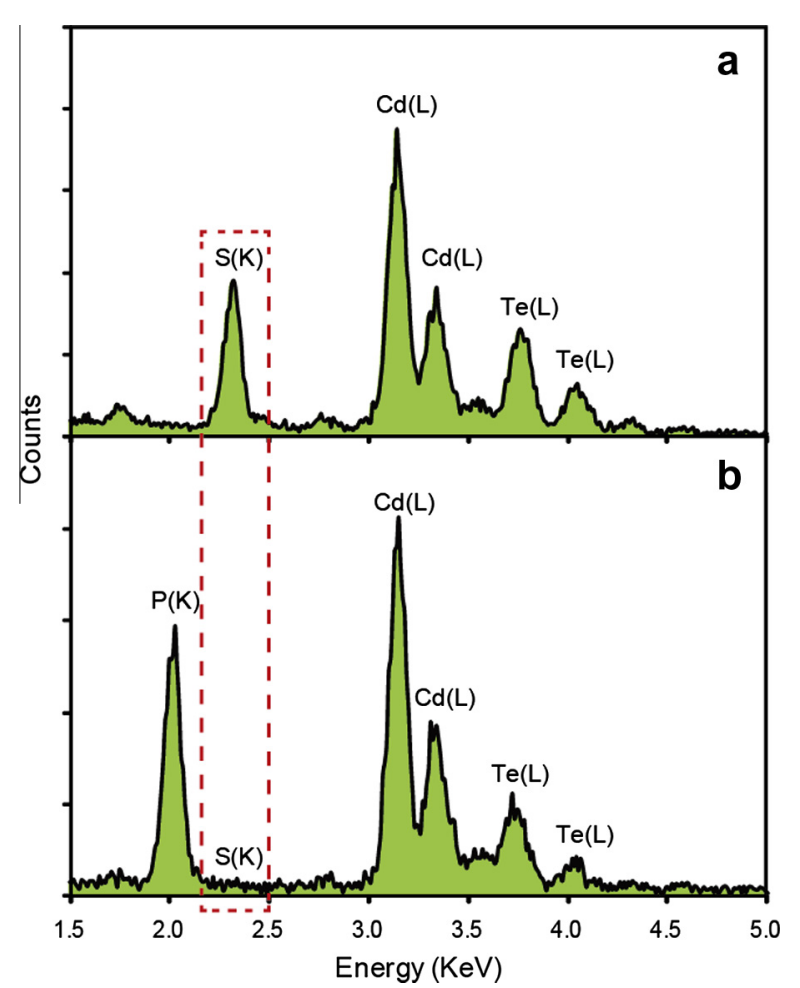

Fig. 3. EDX elemental analysis of CdTe QDs before (a) and after (b) the addition of the FSN-capped gold nanoparticles into CdTe colloidal solution.

attributed to $\mathrm{O}-\mathrm{H}$ stretching vibration of carboxylic acid groups. The bands at $1589 / 1633 \mathrm{~cm}^{-1}$ and $1386 / 1381 \mathrm{~cm}^{-1}$ were the $\mathrm{C}=\mathrm{O}$ asymmetric and symmetric stretching vibrations of carboxylic acid groups of citrate or TGA. The stretching vibration of $\mathrm{C}-\mathrm{O}$ of carboxylic acid groups of citrate or TGA was located at $1175 \mathrm{~cm}^{-1}$ and $1110 \mathrm{~cm}^{-1}$, respectively $[41,42]$. The new-born CdTe QDs showed a new absorbance band at $1066 \mathrm{~cm}^{-1}$, which was attributed to $\mathrm{C}-\mathrm{O}$ stretching vibration of alcoholic hydroxyl group of citrate [43]. However, the TGA-capped CdTe QDs, along with the dialysis solution obtained from the mixture of TGAcapped CdTe QDs and citrate, did not appear the band located at $1066 \mathrm{~cm}^{-1}$. Therefore, we can certainly draw a conclusion that the ligand transformation from TGA to citrate occurred after addition of the FSN-capped gold nanoparticles into the TGA-capped CdTe colloidal solution, and the successful bonding of CdTe QDs with citrate through $-\mathrm{C}-\mathrm{O}-$ functionalities [44]. 


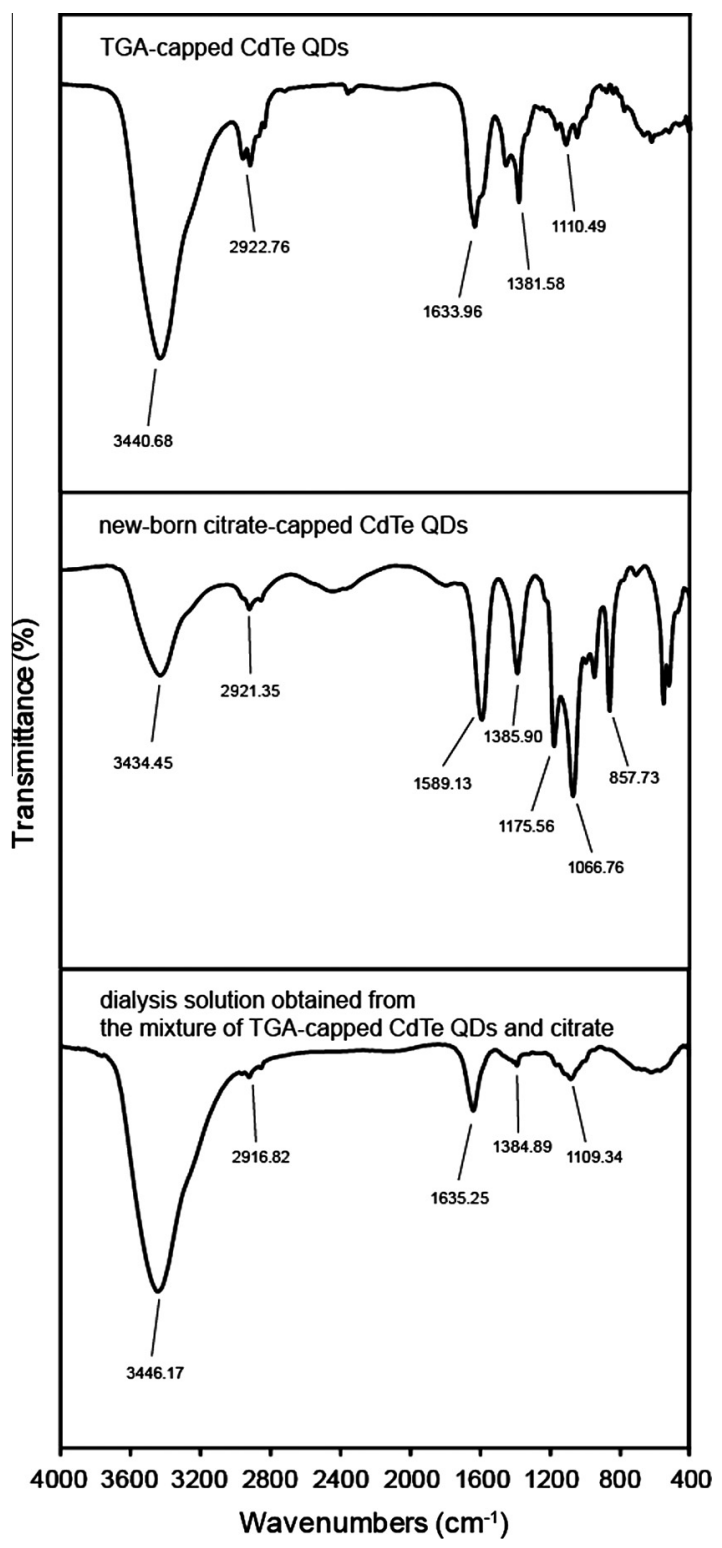

Fig. 4. FT-IR spectra of TGA-capped CdTe QDs, new-born citrate-capped CdTe QDs, dialysis solution obtained from the mixture of TGA-capped CdTe QDs and citrate.

\subsection{Characterization of the new-born CdTe QDs}

The powder XRD profile of the new-born CdTe QDs was compared with that of the original TGA-capped CdTe colloidal solution. Fig. 5a showed broad peaks typical for the original CdTe QDs. Three obvious diffractive peaks were observed which corresponded to the (111), (220) and (311) reflections of cubic zinc blende CdTe [45-47]. After addition of the FSN-capped gold nanoparticles into the original CdTe colloidal solution, the new-born CdTe QDs revealed the same diffraction peaks as the original nanostructures (Fig. 5b). This was further confirmed from the TEM images (inset of Fig. 5). In addition, UV-visible spectra of CdTe QDs before and after the addition of the FSN-capped gold nanoparticles into CdTe colloidal solution kept constant (Fig. S5).

Fig. 6 depicted the fluorescence spectra of the original and newborn CdTe QDs. The spontaneous ligand transformation process seems to have no effect on the fluorescence intensity and fluorescence emission peak, which was supported by the photos of the original and new-born CdTe QDs. The fluorescence quantum yields of the original and new-born CdTe QDs were $18 \%$ and $21 \%$,

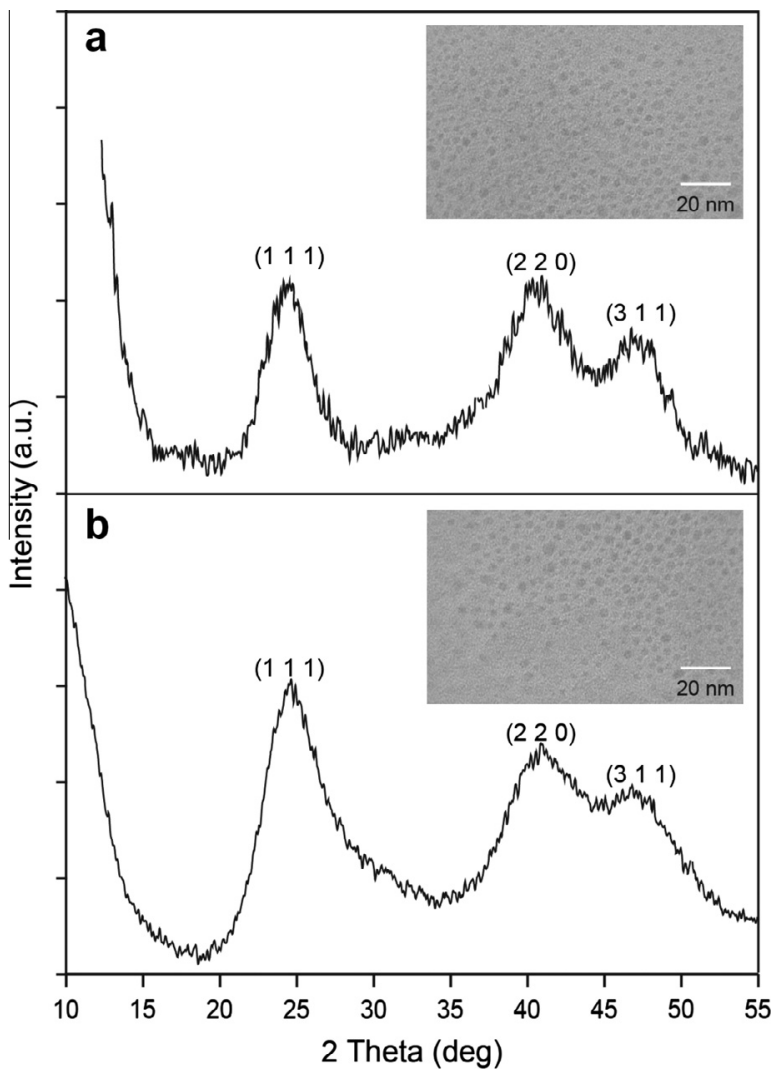

Fig. 5. Powder XRD patterns of CdTe QDs before (a) and after (b) the addition of the FSN-capped gold nanoparticles into CdTe colloidal solution. Inset, TEM images of CdTe QDs before and after the addition of the FSN-capped gold nanoparticles into CdTe colloidal solution.

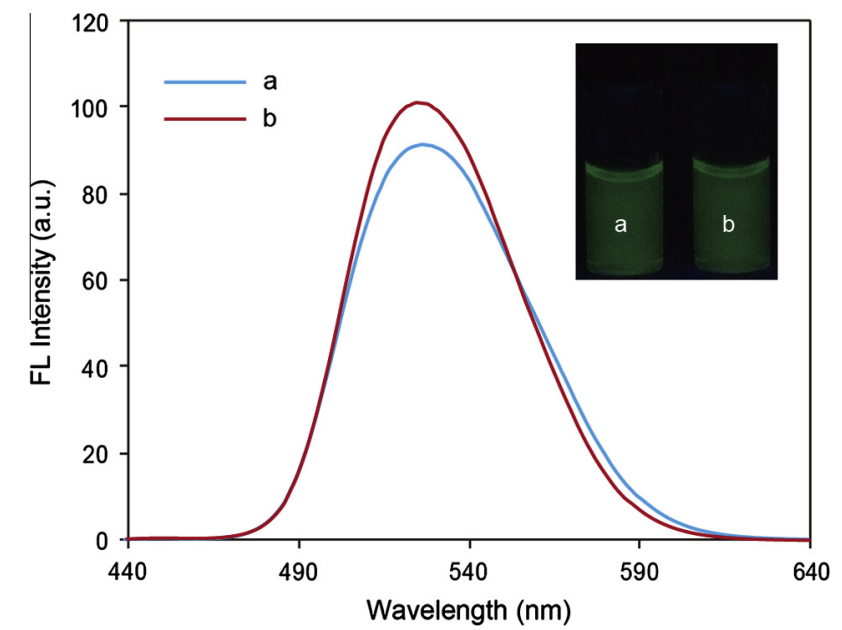

Fig. 6. Fluorescence emission of CdTe QDs before (a) and after (b) the addition of the FSN-capped gold nanoparticles into CdTe colloidal solution. The excitation wavelength was set at $390 \mathrm{~nm}$. Inset, pictures of CdTe QDs before and after the addition of the FSN-capped gold nanoparticles into CdTe colloidal solution.

respectively, which were in good agreement with the value in the literature [48]. In addition, in order to show that this phenomenon is universal, the ligand-exchange reaction between MPA-capped CdTe QDs and FSN-capped gold nanoparticles was also examined. As shown in Fig. S6, after MPA-capped CdTe QDs were exchanged with FSN-capped gold nanoparticles, the fluorescence activity of the new-born CdTe QDs kept stable. 


\subsection{Colloidal stability tests of the new-born citrate-capped CdTe QDs}

The colloidal stability of the new-born citrate-capped QDs was investigated. The results showed that the new-born citrate-capped QD colloidal solution was monodispersed with inconspicuous fluorescence decay at room temperature for at least one week (see Fig. S7). Moreover, the colloidal stability of citrate-capped QDs might be sensitive to $\mathrm{pH}$ and salt concentration in the literature [49]. However, in this work we interestingly found that the newborn citrate-capped QDs exhibited high stability in a wide $\mathrm{pH}$ range and high salt concentration $(600 \mathrm{mM} \mathrm{NaCl})$ (see Fig. S8). In contrast, the new-born citrate-capped QDs obtained by ligand exchange with the citrate-capped gold nanoparticles were easy to aggregate in strongly acidic solutions (Fig. S9). It might be deduced that some FSN ligands in solutions might attain the surface of the new-born citrate-capped QDs by Van der Waals force [50]. Therefore, the new-born citrate-capped QDs might broaden great potential for biological labeling and imaging applications.

\subsection{Cytotoxicity assessment of the new-born CdTe QDs}

The viability of cells was evaluated using MTT assay, based on a cell's ability to metabolize yellow tetrazolium salt into a highly colored formazan product [51-53]. The results were expressed as an average of three nominally identical measurements. As shown in Fig. 7 , the results indicated that severe cytotoxic effects of the TGA-capped CdTe QDs were observed even at a relatively low concentration of the TGA-capped CdTe QDs, along with obvious cell shrinkage. In comparison, at high concentrations of the as-prepared citrate-capped CdTe QDs, the cell viabilities of the citrate-capped CdTe QDs increased to 60-70\% with decreased cell shrinkage, indicating that merely replacing the ligands will not reduce the toxicity as a whole. Note that the extent of cytotoxic effects was correlated with the concentration of original TGAcapped and new-born citrate-capped CdTe QDs, respectively. These interesting results demonstrated that citrate ligands play a key role in reducing the cytotoxicity of CdTe QDs [54]. Accordingly, the as-prepared citrate-capped CdTe QDs might become a very efficient drug delivery vehicle.

\section{Conclusions}

In summary, using FSN-capped gold nanoparticles, we have successfully developed a simple and safe methodology for the preparation of high luminescent citrate-capped CdTe QDs in aqueous solution by highly efficient ligand transformation of CdTe QDs from toxic TGA to green citrate. The mechanism of ligand transformation was verified by UV-visible spectroscopy, powder XRD measurements, TEM images, EDX spectroscopy and FT-IR spectrum. It clearly turned out that TGA ligands on the surface of TGA-capped CdTe QDs can be completely depleted, based on the $\mathrm{Cd}-\mathrm{S}$ bond breaking and the $\mathrm{Au}-\mathrm{S}$ bond formation. In addition, the asprepared citrate-capped CdTe QDs displayed low toxicity against HepG2 cells and a remarkable stability in high salt and a wide $\mathrm{pH}$ range. Our findings may provide a new framework of building green QDs by ligand transformation, which is helpful to overcome the difficulties associated with synthesis of citrate-capped CdTe QDs. It is believed that this concept reported herein could be extended to the green synthesis of a wide range of thiol-capped semiconductor nanocrystals, such as CdS and CdSe.
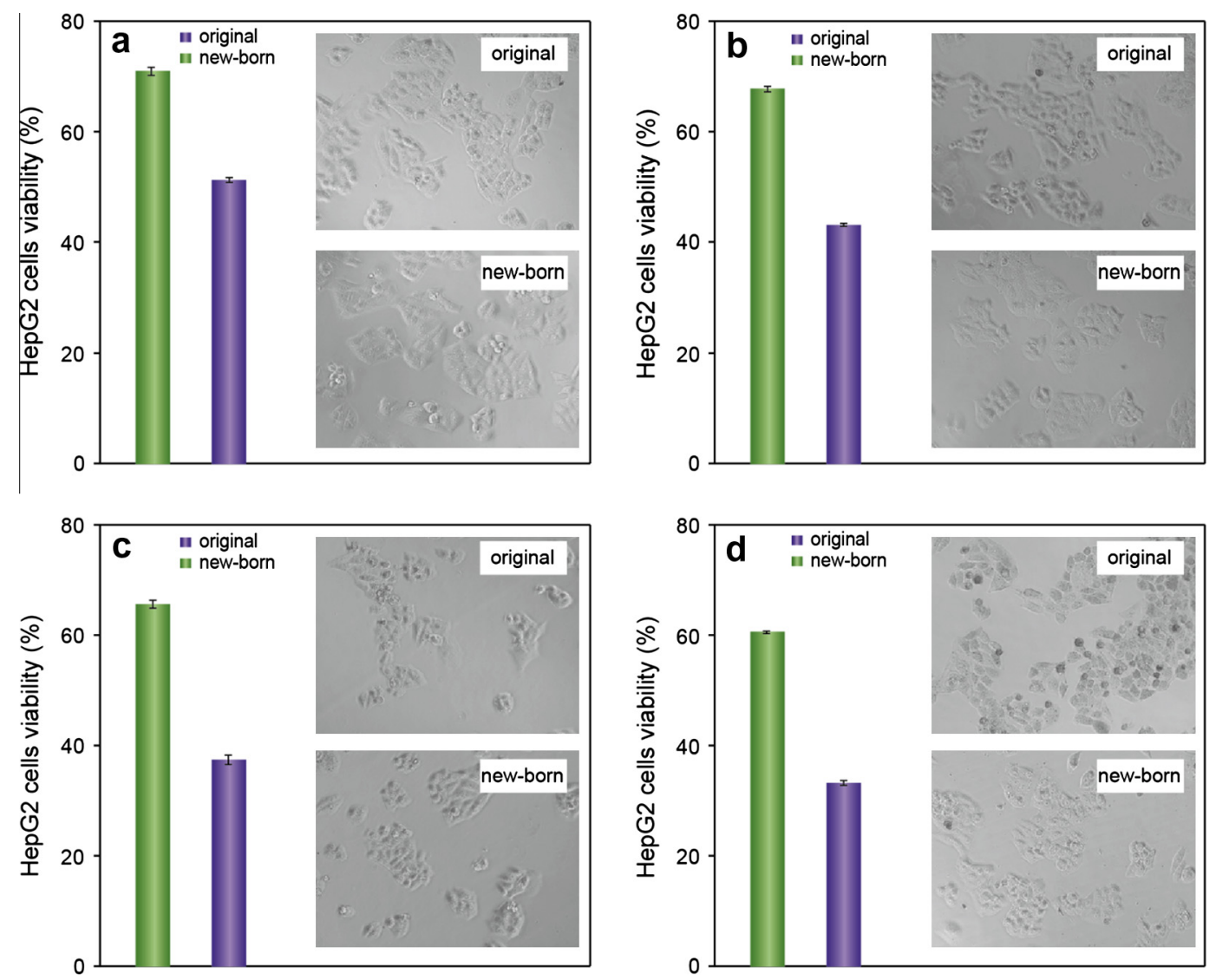

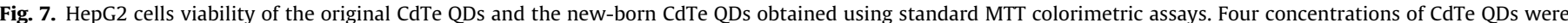

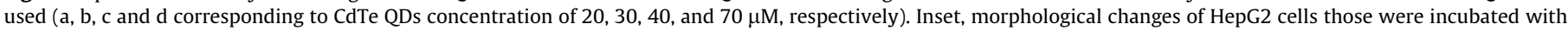
four concentrations of CdTe QDs. 


\section{Acknowledgments}

This work was supported by National Basic Research Program of China (973 Program, 2014CB932103), the National Natural Foundation of China (21077008) and the Program for New Century Excellent Talents in University (NCET-11-0561). We also thank Prof. Jin-Ming Lin, Tsinghua University for his valuable discussions.

\section{Appendix A. Supplementary material}

Supplementary data associated with this article can be found, in the online version, at http://dx.doi.org/10.1016/j.jcis.2013.09.034.

\section{References}

[1] Y. Zhu, Z. Li, M. Chen, H.M. Cooper, G.Q. Lu, Z.P. Xu, J. Colloid Interface Sci. 390 (2013) 3-10.

[2] R. Gill, M. Zayats, I. Willner, Angew. Chem. Int. Ed. 47 (2008) 7602-7625.

[3] M.Q. Chu, X.J. Pan, D. Zhang, Q. Wu, J.L. Peng, W.X. Hai, Biomaterials 33 (2012) $7071-7083$.

[4] Z.A. Peng, X.G. Peng, J. Am. Chem. Soc. 123 (2001) 183-184.

[5] A. Ontam, N. Khaorapapong, M. Ogawa, J. Mater. Chem. 22 (2012) 2000120007.

[6] M.A. Jhonsi, R. Renganathan, J. Colloid Interface Sci. 344 (2010) 596-602.

[7] F. Zhang, E. Lees, F. Amin, P.R. Gil, F. Yang, P. Mulvaney, W.J. Parak, Small 7 (2011) 3113-3127.

[8] A.M. Derfus, W.C.W. Chan, S.N. Bhatia, Nano Lett. 4 (2004) 11-18.

[9] N. Lewinski, V. Colvin, R. Drezek, Small 4 (2008) 26-49.

[10] C.L. Wang, H. Zhang, S.H. Xu, N. Lu, Y. Liu, M.J. Li, H.Z. Sun, J.H. Zhang, B. Yang, J. Phys. Chem. C 113 (2009) 827-833.

[11] P.P. Ingole, R.M. Abhyankar, B.L.V. Prasad, S.K. Haram, Mater. Sci. Eng. B 168 (2010) 60-65.

[12] T. Serrano, I. Gómez, R. Colás, J. Cavazos, Colloids Surf. A 338 (2009) 20-24.

[13] A.L. Rogach, D. Nagesha, J.W. Ostrander, M. Giersig, N.A. Kotov, Chem. Mater. 12 (2000) 2676-2685.

[14] C.L. Li, M. Ando, H. Enomoto, N. Murase, J. Phys. Chem. C 112 (2008) 2019020199.

[15] H. Kim, M. Suh, B.-H. Kwon, D.S. Jang, S.W. Kim, D.Y. Jeon, J. Colloid Interface Sci. 363 (2011) 703-706.

[16] S.J. Tan, N.R. Jana, S.J. Gao, P.K. Patra, J.Y. Ying, Chem. Mater. 22 (2010) 22392247.

[17] E. Woźnica, M.M. Wójcik, M. Wojciechowski, J. Mieczkowski, E. Bulska, K. Maksymiuk, A. Michalska, Anal. Chem 84 (2012) 4437-4442.

[18] J.H. Byeon, J.T. Roberts, Chem. Mater. 24 (2012) 3544-3549.

[19] V. Sashuk, ACS Nano 6 (2012) 10855-10861.

[20] J.S. Kang, T.A. Taton, Langmuir 28 (2012) 16751-16760.

[21] Q.Y. Xiao, H.L. Gao, C. Lu, Q.P. Yuan, Trends Anal. Chem. 40 (2012) 64-76.
22] Y. Tang, J. Yan, X. Zhou, Y. Fu, B. Mao, Langmuir 24 (2008) 13245-13249.

[23] C. Lu, Y.B. Zu, V.W.-W. Yam, Anal. Chem. 79 (2007) 666-672.

[24] C.-C. Huang, W.-L. Tseng, Anal. Chem. 80 (2008) 6345-6350.

[25] J.-H. Lin, C.-W. Chang, Z.-H. Wu, W.-L. Tseng, Anal. Chem. 82 (2010) 87758779.

[26] Y.B. Zu, Z.Q. Gao, Anal. Chem. 81 (2009) 8523-8528.

[27] Y.B. Zu, A.L. Ting, G.S. Yi, Z.Q. Gao, Anal. Chem. 83 (2011) 4090-4094.

[28] L. Li, H.F. Qian, J.C. Ren, Chem. Commun. (2005) 4083-4085.

[29] J.S. Kamal, A. Omari, K.V. Hoecke, Q. Zhao, A. Vantomme, F. Vanhaecke, R.K. Capek, Z. Hens, J. Phys. Chem. C 116 (2012) 5049-5054.

[30] W. Haiss, N.T.K. Thanh, J. Aveyard, D.G. Fernig, Anal Chem. 79 (2007) 4215 4221.

[31] W.W. Yu, L. Qu, W. Guo, X. Peng, Chem. Mater. 15 (2003) 2854-2860.

[32] S. Rucareanu, V.J. Gandubert, R.B. Lennox, Chem. Mater. 18 (2006) 4674-4680.

[33] T. Sen, S. Jana, S. Koner, A. Patra, J. Phys. Chem. C 114 (2010) 19667-19672.

[34] J. Guan, L. Jiang, L.L. Zhao, J. Li, W.S. Yang, Colloid Surf. A - Physicochem. Eng. Asp. 325 (2008) 194-197.

[35] Q.Q. Li, F. Liu, C. Lu, J.-M. Lin, J. Phys. Chem. C 115 (2011) 10964-10970.

[36] K.H. Chen, G.H. Lu, J.B. Chang, S. Mao, K.H. Yu, S.M. Cui, J.H. Chen, Anal. Chem. 84 (2012) 4057-4062.

[37] Z.Y. Tang, Y. Wang, S. Shanbhag, M. Giersig, N.A. Kotov, J. Am. Chem. Soc. 128 (2006) 6730-6736.

[38] Z.Y. Tang, Y. Wang, K. Sun, N.A. Kotov, Adv. Mater. 17 (2005) 358-363.

[39] M.N. Nadagouda, G. Hoag, J. Collins, R.S. Varma, Cryst. Growth Des. 9 (2009) 4979-4983.

[40] G.Z. Shen, C.J. Lee, Cryst. Growth Des. 5 (2005) 1085-1089.

[41] J.J. Ojeda, M.E. Romero-González, S.A. Banwart, Anal. Chem. 81 (2009) 64676473.

[42] J. Sorber, G. Steiner, V. Schulz, M. Guenther, G. Gerlach, R. Salzer, K.-F. Arndt, Anal. Chem. 80 (2008) 2957-2962.

[43] X.N. Lu, C.F. Ross, J.R. Powers, D.E. Aston, B.A. Rasco, J. Agric. Food Chem. 59 (2011) 5215-5221.

[44] M. Hu, H.L. Yu, F.D. Wei, G.H. Xu, J. Yang, Z. Cai, Q. Hu, Spectrochim. Acta A 91 (2012) 130-135

[45] J.J. Liu, Z.X. Shi, Y.C. Yu, R.Q. Yang, S.L. Zuo, J. Colloid Interface Sci. 342 (2010) $278-282$.

[46] M.-R. Chao, Y.-Z. Chang, J.-L. Chen, Biosens. Bioelectron. 42 (2013) 397-402.

[47] S.L. Wu, J. Dou, J. Zhang, S.F. Zhang, J. Mater. Chem. 22 (2012) 14573-14578.

[48] R. Osovsky, A. Shavel, N. Gaponik, L. Amirav, A. Eychmüller, H. Weller, E. Lifshitz, J. Phys. Chem. B 109 (2005) 20244-20250.

[49] G.L. Wang, Y.M. Dong, H.X. Yang, Z.J. Li, Talanta 83 (2011) 943-947.

[50] R.E. Anderson, W.C.W. Chan, ACS Nano 2 (2008) 1341-1352.

[51] R. Klein, M. Kellermeier, D. Touraud, E. Müller, W. Kunz, J. Colloid Interface Sci. 392 (2013) 274-280.

[52] L.G. Bach, M.R. Islam, T.-S. Vo, S.-K. Kim, K.T. Lim, J. Colloid Interface Sci. 394 (2013) 132-140.

[53] S.H. Bhang, N. Won, T.-J. Lee, H. Jin, J. Nam, J. Park, H. Chung, H.-S. Park, Y.-E Sung, S.K. Hahn, B.-S. Kim, S. Kim, ACS Nano 3 (2009) 1389-1398.

[54] B. Dubertret, P. Skourides, D.J. Norris, V. Noireaux, A.H. Brivanlou, A. Libchaber, Science 298 (2002) 1759-1762. 